\title{
Synthesis of 3-fluoroazetidines
}

\author{
Willem Van Brabandt, Guido Verniest, Dirk De Smaele \\ Guillaume Duvey and Norbert De Kimpe* \\ Department of Organic Chemistry, Faculty of Bioscience Engineering, Ghent University, \\ Coupure links 653, B-9000 Ghent, Belgium \\ norbert.dekimpe@UGent.be
}

\section{CONTENTS:}

$\begin{array}{ll}\text { I. General methods } & \text { S2 }\end{array}$

$\begin{array}{ll}\text { II. Synthetic procedures and spectroscopic data } & \text { S2 }\end{array}$

III. ${ }^{1} \mathrm{H}$ and ${ }^{13} \mathrm{C}$ NMR spectra of compounds $\mathbf{4 a - f} \quad$ S8 


\section{General methods}

${ }^{1} \mathrm{H}$ NMR spectra were recorded at 270 or $300 \mathrm{MHz}$ with $\mathrm{CDCl}_{3}$ as solvent and tetramethylsilane as internal standard. ${ }^{13} \mathrm{C}$ NMR spectra were recorded at 68 or $75 \mathrm{MHz}$ with $\mathrm{CDCl}_{3}$ as solvent. Mass spectra were obtained with a mass spectrometer $(70 \mathrm{eV})$ using a $\mathrm{GC}$ MS coupling (20 m glass capillary column, i.d. $0.53 \mathrm{~mm}$, He carrier gas), LC-MS coupling or were recorded using a direct inlet system $(70 \mathrm{eV})$.

\section{Synthetic procedures and spectroscopic data}

\section{Synthesis of $N$-(alkylidene- or 1-arylmethylidene)-2-propenylamines 2a-f}

To a suspension of $0.1 \mathrm{~mol}$ of aldehyde 1 in dry dichloromethane was added 0.15 mol of $\mathrm{MgSO}_{4}$ and $0.1 \mathrm{~mol}$ of 2-methyl or 2-phenyl-2-propenylamine 2. If the amine is used as a hydrochloride salt, $0.1 \mathrm{~mol}$ of triethylamine was added. After 30 minutes of reflux or 1 hour at room temperature (see Scheme 1) the resulting mixture was filtered and the solvent was evaporated, affording $\mathrm{N}$-allylimines $\mathbf{2 a - f}$ (if no triethylamine is used). If triethylamine was used, the reaction mixture was dissolved in dry diethyl ether and triethylamine hydrochloride was filtered off. After evaporation of the solvent, $N$-allylimines 2a-f were obtained, sufficiently pure (>95\%) for further use in the next step. These imines may also be distilled under vacuum. For spectral data see also reference (29).

\section{$N$-(4-Methoxybenzylidene)-2-methyl-2-propenylamine 2c}

Yield: $86 \%$, Bp: $80-83^{\circ} \mathrm{C} / 0.02 \mathrm{mmHg} .{ }^{1} \mathbf{H}$ NMR $\left(270 \mathrm{MHz}, \mathrm{CDCl}_{3}\right): \delta 1.80(3 \mathrm{H}, \mathrm{s}), 3.82$ $(3 \mathrm{H}, \mathrm{s}), 4.11(2 \mathrm{H}, \mathrm{s}), 4.86-4.90(2 \mathrm{H}, \mathrm{m}), 6.92$ and $7.71(4 \mathrm{H}, 2 \times \mathrm{d}, J=8.9 \mathrm{~Hz}), 8.19(1 \mathrm{H}, \mathrm{s}) .{ }^{13} \mathbf{C}$ NMR (68 MHz, $\left.\mathrm{CDCl}_{3}\right): \delta 21.1,55.3,67.0,111.3,113.9,129.7,129.2,143.8,161.2,161.6$. 
IR $\left(\mathrm{NaCl}, \mathrm{cm}^{-1}\right): 1645,1610,1510 . \mathbf{M S}(\mathrm{ES}+) \mathrm{m} / \mathrm{z}(\%): 190\left(\mathrm{M}^{+}+1,100\right)$. Anal. Calcd for $\mathrm{C}_{12} \mathrm{H}_{15} \mathrm{NO}$ : C 76.16; H 7.99; N 7.40. Found: C 76.29; H 7.86; N 7.59.

\section{$N$-(2,2-Dimethyl-1-propylidene)-2-methyl-2-propenylamine 2e}

Yield: 89\%, Bp: 52-56 ${ }^{\circ} \mathrm{C} 41 \mathrm{mmHg} .{ }^{1} \mathbf{H}$ NMR $\left(270 \mathrm{MHz}, \mathrm{CDCl}_{3}\right): \delta 1.09(9 \mathrm{H}, \mathrm{s}) 1.73(3 \mathrm{H}$, s), $3.90(2 \mathrm{H}, \mathrm{s}), 4.81(2 \mathrm{H}, \mathrm{s}), 7.52(1 \mathrm{H}, \mathrm{s}) .{ }^{13} \mathbf{C} \mathbf{N M R}\left(68 \mathrm{MHz}, \mathrm{CDCl}_{3}\right): \delta 21.0,27.0,36.2$, 66.5, 110.8, 143.6, 173.2. IR $\left(\mathrm{NaCl}, \mathrm{cm}^{-1}\right): v_{\mathrm{C}=\mathrm{N}}=1678 . \mathbf{M S}(70 \mathrm{eV}) \mathrm{m} / \mathrm{z}(\%): 139\left(\mathrm{M}^{+}, 0.8\right)$; 125(1); 124(10); 97(3); 84(11); 82(38); 57(17); 56(12); 53(4); 52(4); 41(17). Anal. Calcd for $\mathrm{C}_{9} \mathrm{H}_{17} \mathrm{~N}$ : C 77.63; H 12.31; N 10.06. Found: C 77.76; H 12.19; N 10.01.

\section{Bromofluorination of $N$-(allyl)imines 2 a-f}

For the experimental procedure and spectroscopic data of compound $\mathbf{3 a}$ is referred to the manuscript text.

\section{$N$-(4-Methylbenzylidene)-3-bromo-2-fluoro-2-methylpropylamine 3b}

Yield: 78\%. ${ }^{1} \mathbf{H}$ NMR $\left(270 \mathrm{MHz}, \mathrm{CDCl}_{3}\right): \delta 1.54(3 \mathrm{H}, \mathrm{d}, J=21.5 \mathrm{~Hz}), 2.34(3 \mathrm{H}, \mathrm{s}), 3.60(1 \mathrm{H}$, $\mathrm{d} \times \mathrm{d}, J=16.8 \mathrm{~Hz}, J=11.1 \mathrm{~Hz}), 3.62(1 \mathrm{H}, \mathrm{d} \times \mathrm{d}, J=16.8 \mathrm{~Hz}, J=11.1 \mathrm{~Hz}), 3.73-3.96(2 \mathrm{H}, \mathrm{m})$, 7.18 and $7.62(2 \times 2 \mathrm{H}, 2 \times \mathrm{d}, J=8.1 \mathrm{~Hz}), 8.21(1 \mathrm{H}, \mathrm{s}) .{ }^{13} \mathrm{C}$ NMR $\left(68 \mathrm{MHz}, \mathrm{CDCl}_{3}\right): \delta 21.5$, $22.5(\mathrm{~d}, J=22.0 \mathrm{~Hz}), 37.3(\mathrm{~d}, J=26.8 \mathrm{~Hz}), 66.27(\mathrm{~d}, J=24.5 \mathrm{~Hz}), 94.18(\mathrm{~d}, J=175.8 \mathrm{~Hz})$, 128.2, 129.3, 133.2, 141.2, 163.5. IR $\left(\mathrm{NaCl}, \mathrm{cm}^{-1}\right): 1640 . \mathbf{M S}(70 \mathrm{eV}) \mathrm{m} / \mathrm{z}(\%): 271 / 3\left(\mathrm{M}^{+}, 3\right)$; $192\left(\mathrm{M}^{+}-\mathrm{Br}, 3\right) ; 172(3) ; 163(3) ; 134(3) ; 133(30) ; 132(100) ; 131(6) ; 130(6) ; 118(12) ; 117(9)$; 116(5); 105(99); 104(5); 103(10); 91(11); 90(6); 89(6); 79(8); 77(16); 66(5); 65(12); 63(7); 59(10); 53(7); 52(6); 51(14); 49(5); 44(6). Anal. Calcd for $\mathrm{C}_{12} \mathrm{H}_{15} \mathrm{BrFN}$ : C 52.96; H 5.56; N 5.15. Found: C 53.15; H 5.43; N 5.30. 
$N$-(4-Methoxybenzylidene)-3-bromo-2-fluoro-2-methylpropylamine 3c

Yield: $71 \%$. Boiling point: $135^{\circ} \mathrm{C} / 0.01 \mathrm{~mm} \mathrm{Hg} .{ }^{1} \mathbf{H}$ NMR $\left(270 \mathrm{MHz}, \mathrm{CDCl}_{3}\right): \delta 1.58(3 \mathrm{H}, \mathrm{d}$, $J=21.8 \mathrm{~Hz}), 3.60-3.70(2 \mathrm{H}, \mathrm{m}), 3.84(3 \mathrm{H}, \mathrm{s}), 3.89(2 \mathrm{H}, \mathrm{s}), 6.93$ and $7.72(2 \times 2 \mathrm{H}, 2 \times \mathrm{d}, J=8.9$ $\mathrm{Hz}), 8.24(1 \mathrm{H}, \mathrm{s}) .{ }^{13} \mathrm{C}$ NMR $\left(68 \mathrm{MHz}, \mathrm{CDCl}_{3}\right): \delta 22.6(\mathrm{~d}, J=22.0 \mathrm{~Hz}), 37.27(\mathrm{~d}, J=26.8$ Hz), 55.4, $66.1(\mathrm{~d}, J=24.5 \mathrm{~Hz}), 94.30(\mathrm{~d}, J=203.8 \mathrm{~Hz}), 114.1,130.1,128.6,162.1,163.3 . \mathbf{I R}$ $\left(\mathrm{NaCl}, \mathrm{cm}^{-1}\right): 1606,1310,1265,1525,1170,1035 . \mathrm{MS}(\mathrm{ES}+) \mathrm{m} / \mathrm{z}(\%): 288 / 90\left(\mathrm{M}^{+}+1,100\right)$. Anal. Calcd for $\mathrm{C}_{12} \mathrm{H}_{15}$ BrFNO: C 50.02; H 5.25; N 4.86. Found: C 50.23; H 5.39; N 4.78.

\section{$N$-(2-Ethylbutyl)-3-bromo-2-fluoro-2-methylpropylamine 3d}

Yield: $68 \%$. Boiling point: $70-75^{\circ} \mathrm{C} / 0.3 \mathrm{~mm} \mathrm{Hg} .{ }^{1} \mathbf{H} \mathbf{N M R}\left(270 \mathrm{MHz}, \mathrm{CDCl}_{3}\right): \delta 0.89(6 \mathrm{H}, \mathrm{t}$, $J=7.6 \mathrm{~Hz}), 1.43-1.54(4 \mathrm{H}, \mathrm{m}), 1.54(3 \mathrm{H}, \mathrm{d}, J=21.5 \mathrm{~Hz}), 2.01-2.19(1 \mathrm{H}, \mathrm{m}), 3.51-3.60(1 \mathrm{H}$, m), 3.62-3.72 (2H, m), $7.47(1 \mathrm{H}, \mathrm{d} \times \mathrm{t}, J=6.6 \mathrm{~Hz}, J=1.1 \mathrm{~Hz}) .{ }^{13} \mathbf{C} \mathbf{N M R}\left(68 \mathrm{MHz}, \mathrm{CDCl}_{3}\right): \delta$ 11.6, $22.5(\mathrm{~d}, J=23.2 \mathrm{~Hz}), 24.9,37.0(\mathrm{~d}, J=28.1 \mathrm{~Hz}), 48.48,66.5(\mathrm{~d}, J=23.2 \mathrm{~Hz}), 94.1(\mathrm{~d}, J$ $=175.8 \mathrm{~Hz}), 172.9 . \mathbf{I R}\left(\mathrm{NaCl}, \mathrm{cm}^{-1}\right): 2975,2939,2882,1671,1463,1387 . \mathbf{M S}(\mathrm{ES}+) \mathrm{m} / \mathrm{z}$ (\%): 252/4 $\left(\mathrm{M}^{+}+1,100\right)$. Anal. Calcd for $\mathrm{C}_{10} \mathrm{H}_{19} \mathrm{BrFN}$ : $\mathrm{C} 47.63 ; \mathrm{H} 7.59 ; \mathrm{N} 5.55$. Found: $\mathrm{C}$ $47.53 ; \mathrm{H} 7.48 ; \mathrm{N} 5.68$.

\section{$N$-(2,2-Dimethyl-1-propylidene)-3-bromo-2-fluoro-2-methylpropylamine 3e}

Yield: $73 \% .{ }^{1} \mathbf{H}$ NMR $\left(270 \mathrm{MHz}, \mathrm{CDCl}_{3}\right): \delta 1.07(9 \mathrm{H}, \mathrm{s}), 1.50(3 \mathrm{H}, \mathrm{d}, J=21.5 \mathrm{~Hz}), 3.51-3.73$ $(4 \mathrm{H}, \mathrm{m}), 7.58(1 \mathrm{H}, \mathrm{d} \times \mathrm{d}, J=1.0 \mathrm{~Hz}, J=1.3 \mathrm{~Hz}) \cdot{ }^{13} \mathbf{C} \mathbf{N M R}\left(68 \mathrm{MHz}, \mathrm{CDCl}_{3}\right): \delta 22.5(\mathrm{~d}, J=$ $23.2 \mathrm{~Hz}), 26.7,36.4,36.9(\mathrm{~d}, J=28.0 \mathrm{~Hz}), 66.1(\mathrm{~d}, J=24.5 \mathrm{~Hz}), 94.1(\mathrm{~d}, J=175.8 \mathrm{~Hz})$, 175.4. IR $\left(\mathrm{NaCl}, \mathrm{cm}^{-1}\right)$ : 1671. MS (ES+) m/z (\%): 237/9 (M+1), 222/4 (1), 180/2(16), 153/5(19), 133/5(5), 98(100), 84(5), 82(7), 73(42), 69(21), 59(8), 57(13), 56(12), 55(13), 
53(13), 47(10), 42(18), 41(48). Anal. Calcd for $\mathrm{C}_{9} \mathrm{H}_{17} \mathrm{BrFN}$ : C 45.39; H 7.20; N 5.88. Found: C 45.31; H 7.34; N 5.95.

\section{$N$-Benzylidene-3-bromo-2-fluoro-2-phenylpropylamine $3 f$}

Yield: $77 \% .{ }^{1} \mathbf{H}$ NMR $\left(270 \mathrm{MHz}, \mathrm{CDCl}_{3}\right): \delta 3.96(1 \mathrm{H}, \mathrm{d} \times \mathrm{d}, J=20.4 \mathrm{~Hz}, J=11.4 \mathrm{~Hz}), 4.09$

$(1 \mathrm{H}, \mathrm{d} \times \mathrm{d}, J=20.4 \mathrm{~Hz}, J=11.4 \mathrm{~Hz}), 4.13-4.20(2 \mathrm{H}, \mathrm{m}), 7.26-7.87(10 \mathrm{H}, \mathrm{m}), 8.26(1 \mathrm{H}, \mathrm{s}) .{ }^{13} \mathrm{C}$

NMR (68 MHz, $\left.\mathrm{CDCl}_{3}\right): \delta 37.3(\mathrm{~d}, J=25.6 \mathrm{~Hz}), 67.3(\mathrm{~d}, J=26.8 \mathrm{~Hz}), 96.2(\mathrm{~d}, J=176.4 \mathrm{~Hz})$, $124.9,125.0,128.3,128.3,128.6,131.1,135.2,139.3(\mathrm{~d}, J=201.6 \mathrm{~Hz}), 164,4 . \mathbf{I R}\left(\mathrm{NaCl}, \mathrm{cm}^{-}\right.$

$\left.{ }^{1}\right)$ : 1643. MS (ES+) m/z (\%): decomposition of the product occurred during direct inlet or GC-MS. Anal. Calcd for $\mathrm{C}_{16} \mathrm{H}_{15} \mathrm{BrFN}$ : C 60.02; H 4.72; N 4.37. Found: $\mathrm{C} 60.19 ; \mathrm{H}$ 4.66; N 4.19 .

\section{Synthesis of 3-fluoroazetidines 4a-f}

For the experimental procedure and spectroscopic data of compound $\mathbf{4 a}$ is referred to the manuscript text.

\section{3-Fluoro-3-methyl-1-(4-methylbenzyl)azetidine 4b}

Yield: $61 \% .{ }^{1} \mathbf{H}$ NMR $\left(270 \mathrm{MHz}, \mathrm{CDCl}_{3}\right): \delta 1.55(3 \mathrm{H}, \mathrm{d}, J=22.1 \mathrm{~Hz}), 2.30(3 \mathrm{H}, \mathrm{s}), 3.20(2 \mathrm{H}$, $\mathrm{d} \times \mathrm{d}, J=22.1 \mathrm{~Hz}, J=8.3 \mathrm{~Hz}), 3.29(2 \mathrm{H}, \mathrm{d} \times \mathrm{d} \times \mathrm{d}, J=13.8 \mathrm{~Hz}, J=8.3 \mathrm{~Hz}, J=2.0 \mathrm{~Hz}), 3.58$ $(2 \mathrm{H}, \mathrm{s}), 7.07-7.19$ (4H, m). ${ }^{13} \mathrm{C}$ NMR $\left(68 \mathrm{MHz}, \mathrm{CDCl}_{3}\right): \delta 21.0,23.0(\mathrm{~d}, J=25.6 \mathrm{~Hz}), 63.2$, $65.1(\mathrm{~d}, J=21.9 \mathrm{~Hz}), 89.7(\mathrm{~d}, J=203.9 \mathrm{~Hz}), 128.4,129.0,134.6,136.7 . \mathbf{I R}\left(\mathrm{NaCl}, \mathrm{cm}^{-1}\right)$ : 1517, 1381, 1361, 1327, 1230, 1212. MS (70 eV) m/z (\%): $193\left(\mathrm{M}^{+}, 1\right) ; 192(7) ; 191(3)$; 178(1); 149(1); 132(7); 112(17); 106(17); 105(100); 104(3); 103(5); 102(3); 91(3); 79(8); 78(5); 77(13); 44(20); 43(1); 42(3); 41(7). Anal. Calcd for $\mathrm{C}_{12} \mathrm{H}_{16} \mathrm{FN}$ : C 74.58; H 8.34; N 7.25. Found: C 74.76; H 8.45; N 7.12. 


\section{3-Fluoro-3-methyl-1-(4-methoxybenzyl)azetidine 4c}

Yield: $65 \% .{ }^{1} \mathbf{H}$ NMR $\left(270 \mathrm{MHz}, \mathrm{CDCl}_{3}\right): \delta 1.59(3 \mathrm{H}, \mathrm{d}, J=22.4 \mathrm{~Hz}), 3.17-3.38(4 \mathrm{H}, \mathrm{m})$, $3.61(2 \mathrm{H}, \mathrm{s}), 3.80(3 \mathrm{H}, \mathrm{s}), 6.85$ and $7.20(4 \mathrm{H}, 2 \times \mathrm{d}, J=9.2 \mathrm{~Hz}) .{ }^{13} \mathbf{C} \mathbf{N M R}\left(68 \mathrm{MHz}, \mathrm{CDCl}_{3}\right): \delta$ $23.2(\mathrm{~d}, J=25.6 \mathrm{~Hz}), 55.2,62.2,65.1(\mathrm{~d}, J=21.9 \mathrm{~Hz}), 89.9(\mathrm{~d}, J=203.8 \mathrm{~Hz}), 113.8,129.7$, 129.9, 158.8. IR (NaCl, $\left.\mathrm{cm}^{-1}\right): 1511,1304,1259,1248,1180,1037 . \mathbf{M S}(\mathrm{ES}+) \mathrm{m} / \mathrm{z}(\%): 211$ $\left(\mathrm{M}^{+}+2,100\right)$. Anal. Calcd for $\mathrm{C}_{12} \mathrm{H}_{16} \mathrm{FNO}$ : $\mathrm{C} 68.88 ; \mathrm{H}$ 7.71; $\mathrm{N}$ 6.69. Found: C 68.62; H 7.56; N 6.79 .

\section{3-Fluoro-3-methyl-1-(2-ethylbutyl)azetidine 4d}

Yield: $61 \% .{ }^{1} \mathbf{H}$ NMR $\left(300 \mathrm{MHz}, \mathrm{CDCl}_{3}\right): \delta 0.84(6 \mathrm{H}, \mathrm{t}, J=7.3 \mathrm{~Hz}), 1.15-1.39(5 \mathrm{H}, \mathrm{m}), 1.58$ $(3 \mathrm{H}, \mathrm{d}, J=22.3 \mathrm{~Hz}), 2.36(2 \mathrm{H}, \mathrm{d}, J=6.3 \mathrm{~Hz}), 3.08(2 \mathrm{H}, \mathrm{m}), 3.32(2 \mathrm{H}, \mathrm{d} \times \mathrm{d} \times \mathrm{d}, J=13.2 \mathrm{~Hz}, J$ $=7.0 \mathrm{~Hz}, J=2.1 \mathrm{~Hz}) .{ }^{13} \mathrm{C} \mathbf{N M R}\left(68 \mathrm{MHz}, \mathrm{CDCl}_{3}\right): \delta 10.9,23.2(\mathrm{~d}, J=25.4 \mathrm{~Hz}), 24.0,39.6$, 63.9, $66.1(\mathrm{~d}, J=20.8 \mathrm{~Hz}), 89.9(\mathrm{~d}, J=203.1 \mathrm{~Hz}) . \mathbf{I R}\left(\mathrm{NaCl}, \mathrm{cm}^{-1}\right): 1455,1380,1054 . \mathbf{M S}$ $(\mathrm{ES}+) \mathrm{m} / \mathrm{z}(\%): 175\left(\mathrm{M}^{+}+2,100\right)$. Anal. Calcd for $\mathrm{C}_{10} \mathrm{H}_{20} \mathrm{FN}: \mathrm{C} 69.32 ; \mathrm{H} 11.63 ; \mathrm{N} 8.08$. Found: C 69.45; H 11.78; N 7.95.

\section{3-Fluoro-3-methyl-1-(2,2-dimethylpropyl)azetidine 4e}

Yield: 59\%. ${ }^{1} \mathbf{H}$ NMR $\left(270 \mathrm{MHz}, \mathrm{CDCl}_{3}\right): \delta 0.86(9 \mathrm{H}, \mathrm{s}), 1.58(3 \mathrm{H}, \mathrm{d}, J=22.4 \mathrm{~Hz}), 2.26(2 \mathrm{H}$, s), 3.19-3.43 (4H, m). ${ }^{13} \mathbf{C}$ NMR (68 MHz, $\left.\mathrm{CDCl}_{3}\right): \delta 20.1,23.7(\mathrm{~d}, J=25.6 \mathrm{~Hz}), 32.4,68.1$ $(\mathrm{d}, J=20.7 \mathrm{~Hz}), 73.2,90.3(\mathrm{~d}, J=202.6 \mathrm{~Hz}) . \mathbf{I R}\left(\mathrm{NaCl}, \mathrm{cm}^{-1}\right): 1378,1358,1322,1229,1205$, 1067. MS (70 eV) m/z (\%): $159\left(\mathrm{M}^{+} ; 3\right) ; 144(6) ; 102(100) ; 82(6) ; 73(4) ; 57(7) ; 55(8) ; 43(21)$; 42(37); 41(12). Anal. Calcd for $\mathrm{C}_{9} \mathrm{H}_{18} \mathrm{FN}$ : C 67.88; $\mathrm{H}$ 11.39; $\mathrm{N}$ 8.80. Found: $\mathrm{C} 67.76 ; \mathrm{H}$ $11.55 ; \mathrm{N} 8.85$.

\section{1-Benzyl-3-fluoro-3-phenylazetidine 4f}


Yield: 60\%. ${ }^{1} \mathbf{H}$ NMR $\left(270 \mathrm{MHz}, \mathrm{CDCl}_{3}\right): \delta 3.60-3.79(4 \mathrm{H}, \mathrm{m}), 3.82(2 \mathrm{H}, \mathrm{s}), 7.29-7.61(10 \mathrm{H}$, m). ${ }^{13}$ C NMR (68 MHz, $\left.\mathrm{CDCl}_{3}\right): \delta 63.3,66.2(\mathrm{~d}, J=23.2 \mathrm{~Hz}), 91.7(\mathrm{~d}, J=206.2 \mathrm{~Hz}), 124.2$, 124.4, 127.4, 128.2, 128.4, 140.1, 138.9 (d, $J=199.0 \mathrm{~Hz}) . \mathbf{I R}\left(\mathrm{NaCl}, \mathrm{cm}^{-1}\right): 1488,1445,1292$, 1210, 1050, 1023. MS (70 eV) m/z (\%): $241\left(\mathrm{M}^{+} ; 7\right) ; 220(2) ; 199(2) ; 130(2) ; 122(47)$; 119(4); 118(9); 96(4); 92(10); 91(100); 77(3); 65(6); 51(4); 42(4); 41(5). Anal. Calcd for $\mathrm{C}_{16} \mathrm{H}_{16} \mathrm{FN}$ : C 79.64; H 6.68; N 5.80. Found: C 79.75; H 6.60; N 5.86.

\section{1-Benzhydryl-2-(fluoromethyl)aziridine 10}

Yield: $17 \% .{ }^{1} \mathbf{H}$ NMR $\left(270 \mathrm{MHz}, \mathrm{CDCl}_{3}\right): \delta 1.64(1 \mathrm{H}, \mathrm{d} \times \mathrm{d}, J=6.6 \mathrm{~Hz}, J=2.3 \mathrm{~Hz}), 1.88$ $(1 \mathrm{H}, \mathrm{d} \times \mathrm{d}, J=3.3 \mathrm{~Hz}, J=1.0 \mathrm{~Hz}), 1.94-2.03(1 \mathrm{H}, \mathrm{m}), 3.58(1 \mathrm{H}, \mathrm{s}), 4.23-4.33$ and $4.41-4.51$ $(2 \mathrm{H}, \mathrm{m}), 7.20-7.49(10 \mathrm{H}, \mathrm{m}) .{ }^{13} \mathbf{C} \mathbf{N M R}\left(68 \mathrm{MHz}, \mathrm{CDCl}_{3}\right): \delta 31.67(\mathrm{~d}, J=6.1 \mathrm{~Hz}), 38.18(\mathrm{~d}, J$ $=26.8 \mathrm{~Hz}), 77.7,85.04(\mathrm{~d}, J=168.5 \mathrm{~Hz}), 127.2,127.2,127.3,127.5,128.4,128.4 . \mathbf{I R}(\mathrm{NaCl}$, $\left.\mathrm{cm}^{-1}\right): 1492 ; 1451 ; 1078 ; 1055 ; 1028 ; 1000 . \mathbf{M S}(70 \mathrm{eV}) \mathrm{m} / \mathrm{z}(\%): 241\left(\mathrm{M}^{+} ; 3\right) ; 168(15) ; 167$ (100). Anal. Calcd for $\mathrm{C}_{16} \mathrm{H}_{16} \mathrm{FN}$ : C 79.64; H 6.68; N 5.80. Found: C 79.71; H 6.63; N 5.76. 

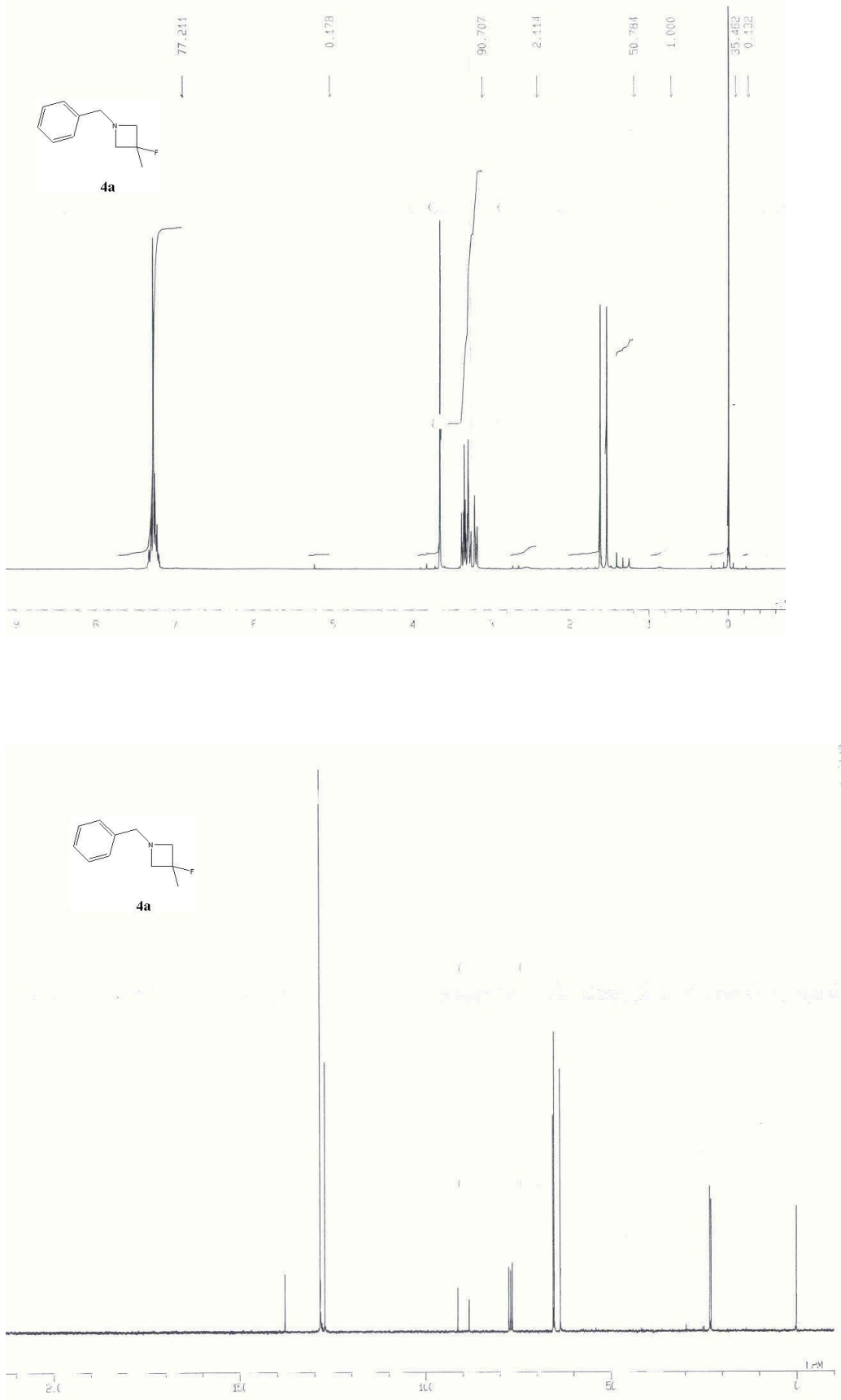

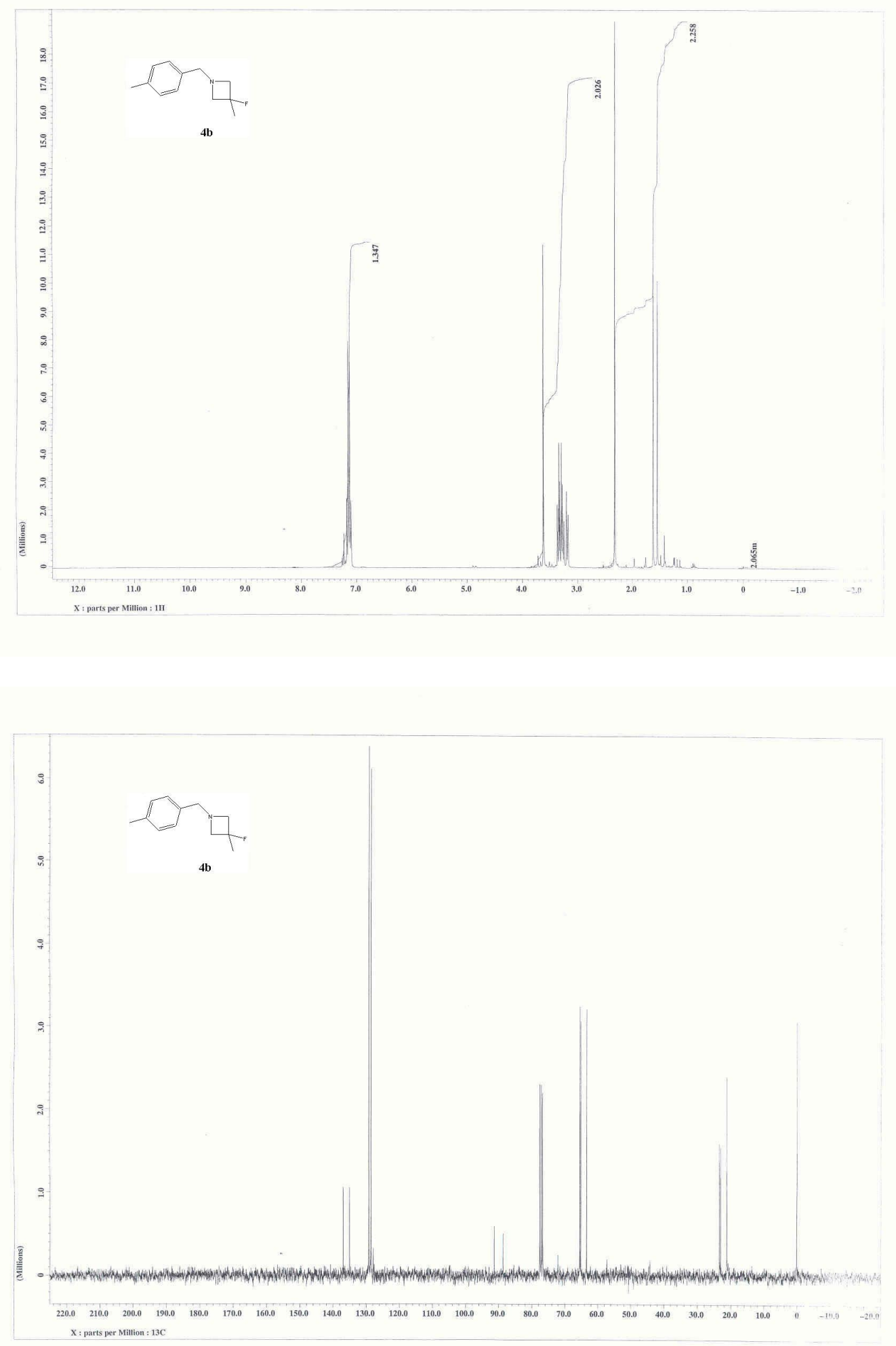

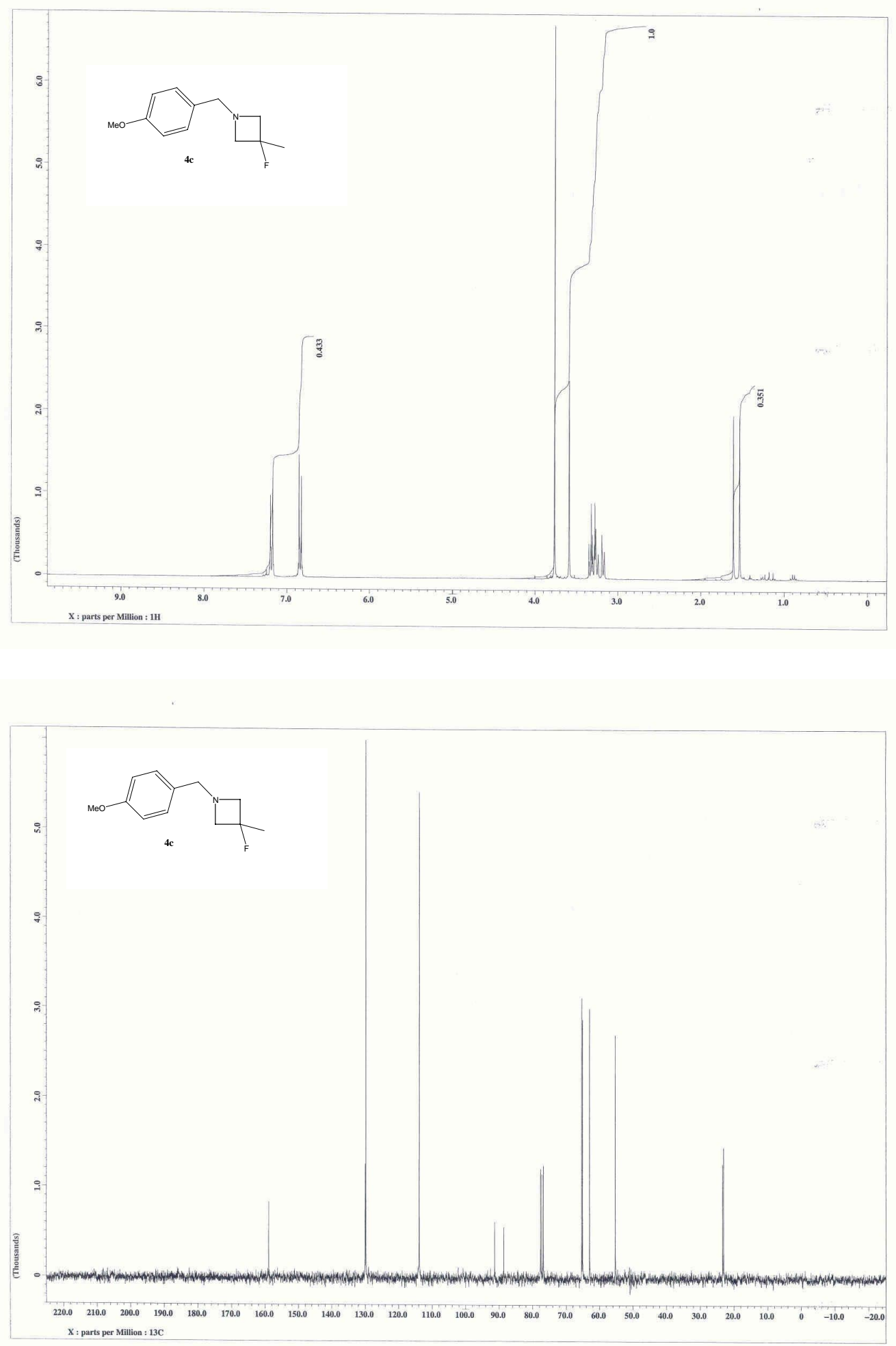

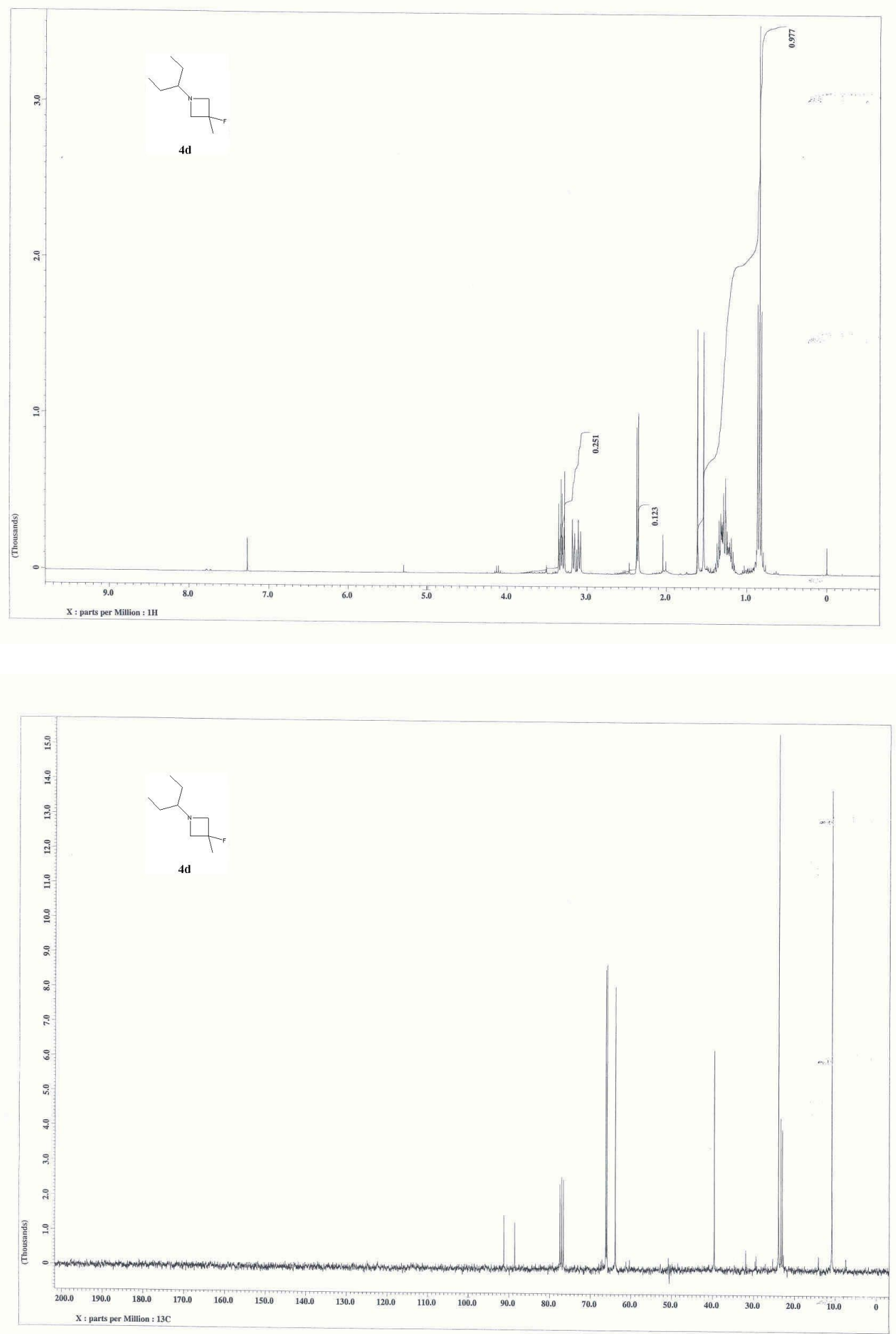


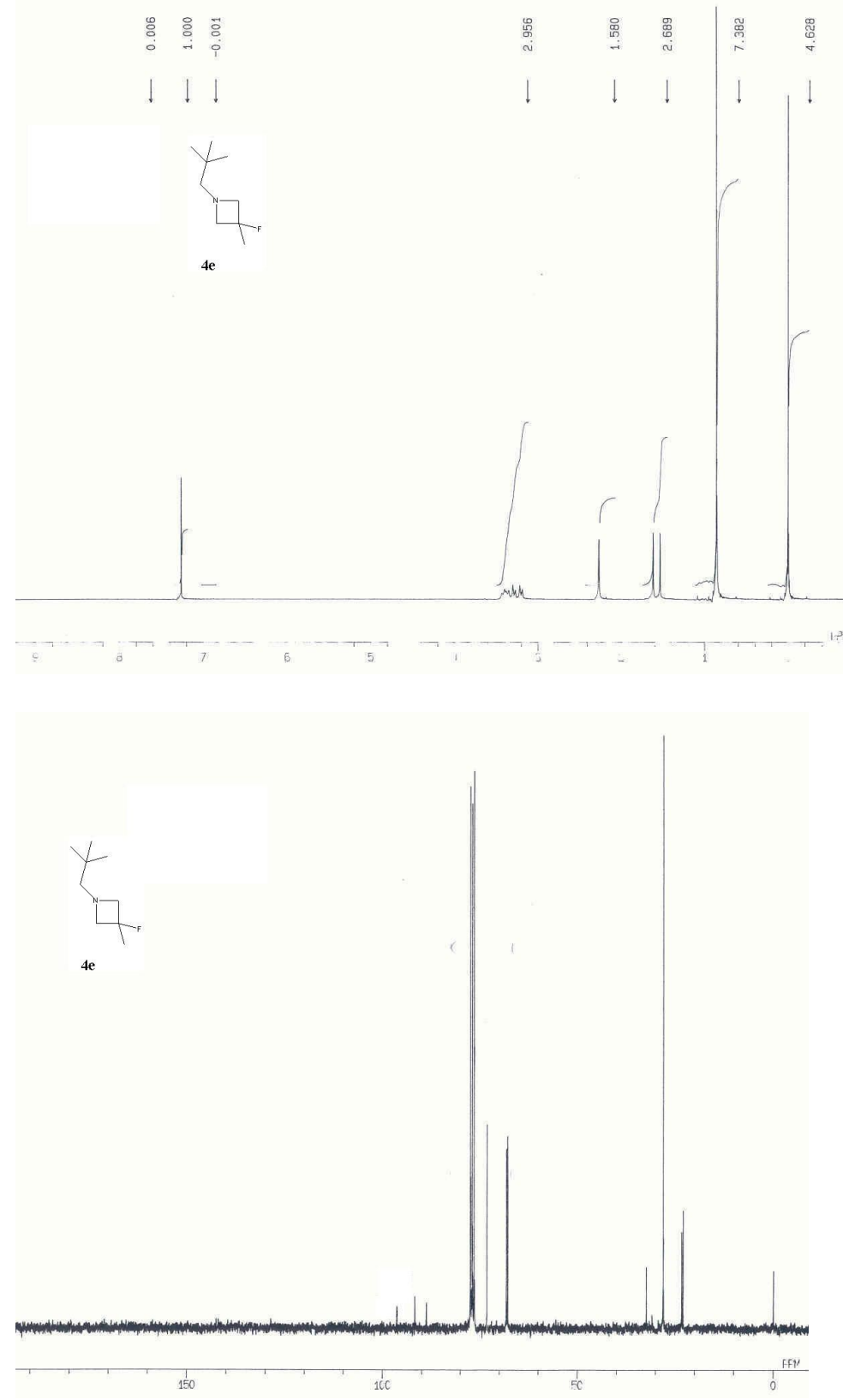



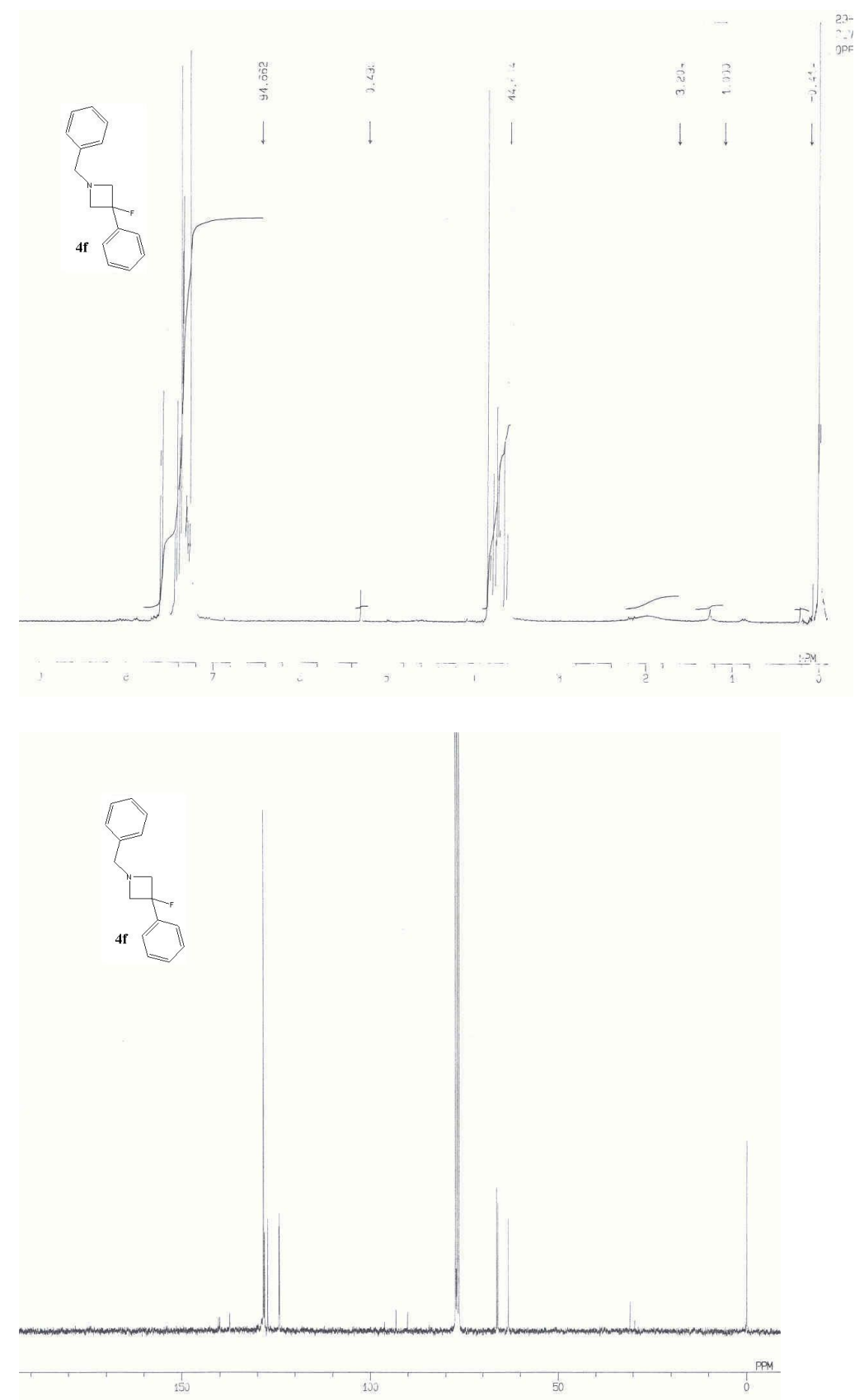